\title{
An FDTD/MoM Hybrid Technique for Modeling Complex Antennas in the Presence of Heterogeneous Grounds
}

\author{
Zhubo Huang, Member, IEEE, Kenneth R. Demarest, Senior Member, IEEE, and Richard G. Plumb, Senior Member, IEEE
}

\begin{abstract}
Calculating the current distribution and radiation patterns for ground-penetrating radar antennas is a challenging problem because of the complex interaction between the antenna, the ground, and any buried scatterer. Typically, numerical techniques that are well suited for modeling the antennas themselves are not well suited for modeling the heterogeneous grounds, and visa versa. For example the finite-difference time-domain (FDTD) technique is well suited for modeling fields in heterogeneous media, whereas the method of moments (MoM) is well suited for modeling complex antennas in free space. This paper describes a hybrid technique, based upon the equivalence principle, for calculating an antenna's current distribution radiation pattern when the antenna is located near an air-ground interface.

The original problem is decomposed into two coupled equivalent problems: one for the antenna geometry and the other for the ground geometry, with field information passing between them via a rapidly converging iterative procedure. The fields in each region may be modeled using numerical techniques best suited to them. Results for several test cases are presented, using FDTD to model the ground problem and MoM for the antenna problem, that demonstrate the accuracy of this hybrid technique.
\end{abstract}

Index Terms - Antenna radiation patterns, finite difference methods, ground, iterative methods, radar.

\section{INTRODUCTION}

G ROUND-PENETRATING radars (GPR's) present a number of unique challenges to electromagnetic modelers. Most of these challenges are in one way or another related to the ground itself, which has a significant impact on the signals that are transmitted and received by GPR's. Since the ground usually is a lossy dielectric, integral-equation techniques such as the method of moments are not well suited for these geometries. This is because they would require either the use of a complicated Green's function or an enormous number of unknowns for modeling the ground.

One technique that has shown itself to be well suited to a number of the special problems imposed by GPR's is the finitedifference time-domain (FDTD) technique [1]. Since FDTD is a partial differential-equation technique, the presence of a ground does not seriously impact the number of unknowns that must be determined. This technique has been used for a number of years to model GPR's whose antennas are located either close to [2], [3] or far from the air-ground interface [4],

Manuscript received December 26, 1997; revised November 10, 1998. The authors are with the Remote Sensing Laboratory, University of Kansas, Lawrence, KS 66045-2969 USA (e-mail: demarest@eecs.ukans.edu).

Publisher Item Identifier S 0196-2892(99)06275-0.
[5]. During this period, a number of improvements to FDTD have been developed to handle several special difficulties generic to GPR modelers. Examples include the ability to model stratified grounds for plane-wave incident fields [6], a near-to-far-field transformation for buried scatterers [4], and improved absorbing-boundary conditions.

In spite of these strides in adapting FDTD for GPR modeling, one important modeling problem has remained: the modeling of complex GPR antennas. Although FDTD is well suited for modeling simple antennas such as dipoles, it is not as well suited for modeling antennas that are not easily resolved by a rectangular grid. More complicated antennas involving wires and surfaces extended at nonorthogonal angles can be modeled by either the use of subgrids or curved-surface formalisms [7], [8]. However, these techniques tend to make most FDTD codes more numerically intensive, while still not providing the kind of accuracy routinely found with integral equation techniques such as the method of moments (MoM) [9].

In this paper, we describe a hybrid technique that combines FDTD and MoM to model GPR's with complex antennas. This technique uses the equivalence principle to separate a GPR geometry into two subgeometries that are easily modeled using FDTD and MoM, respectively. A rapidly converging iterative technique then passes boundary-field information between these subgeometries, resulting in a full-wave solution that correctly models the performance of the antenna in the presence of the ground. Numerical results are presented that demonstrate the accuracy of this technique.

\section{HYBRID TECHNIQUE}

Consider the typical GPR geometry shown in Fig. 1. Here, an antenna is located above a ground that contains a buried object. To invoke the Schelkunoff equivalence principle [10], an imaginary closed surface $S$ (Huygens' surface) with an outward normal vector $n$ is placed around the antenna. Two coupled-equivalent problems are established: one problem equivalent to the original problem internal to $S$ and the other equivalent to the original problem external to $S$, as shown in Fig. 1.

The internal problem (antenna geometry) contains the original antenna geometry, the original fields inside $S$, and the null fields outside $S$. Support of this field configuration requires that surface currents $-J_{S}$ and $-M_{S}$ be impressed on $S$ in 


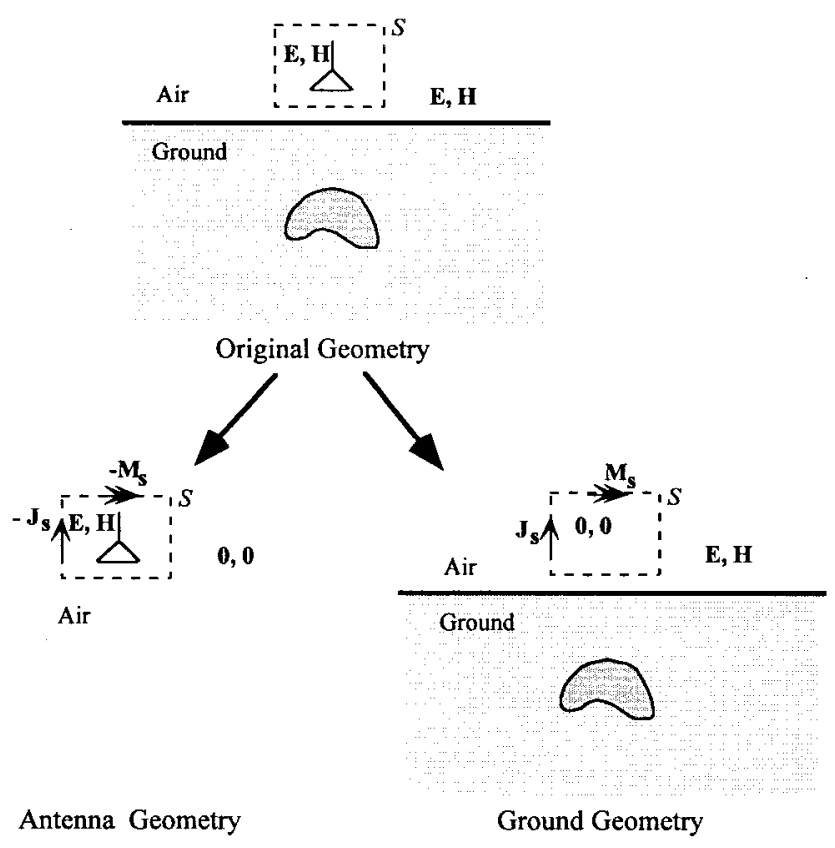

Fig. 1. Decomposition of an original GPR geometry into two subgeometries: antenna geometry and ground geometry.

addition to the original impressed sources on the antenna, where

$$
\begin{aligned}
\boldsymbol{J}_{S} & =\boldsymbol{n} \times \boldsymbol{H} \\
\boldsymbol{M}_{S} & =\boldsymbol{E} \times \boldsymbol{n} .
\end{aligned}
$$

Similarly, the external problem (ground geometry) contains the original ground geometry, the original fields external to $S$, and the null fields inside $S$. To support the fields for this configuration requires surface currents $J_{S}$ and $M_{S}$ be impressed on $S$, where $\boldsymbol{E}$ and $\boldsymbol{H}$ are the electric and magnetic fields along $S$ in the original problem. In the discussion that follows, we will assume that the antenna does not contact the ground, so that the Huygens' surface does not cross the air-ground boundary.

Dividing the original geometry shown in Fig. 1 into two subgeometries has the obvious advantage that the ground geometry does not contain the antenna, and the antenna geometry does not contain the ground and buried scatterer. However, the cost of this split is that each subgeometry has unknown surface currents that must somehow be found in order to model either or both subgeometries. There are a number of ways in which these currents can be handled, but they generally can be classed either as direct or iterative techniques.

Direct solution techniques typically are methods whereby the exact solution is obtained using a fixed number of operations. Solving a linear system of equations using direct-matrix integration is an example. For this two-subgeometry problem, a direct solution would require that the unknown currents on the Huygens' surface be represented as unknowns in both subgeometries. One example of this kind of scheme is found in [11], where the MoM was used on two subgeometries bounded by a small aperture, resulting in a large system of equations.
As attractive as direct problem solvers are, however, we chose to use an iterative technique to solve for the transmitted and scattered fields in our GPR modeler. There were two reasons for this choice. The first is that the addition of electric and magnetic current unknowns along the Huygens' surface would add a large number of unknowns to the antenna geometry. Unlike [11], where the bounding surface between the two regions was electrically small, the Huygens' surface in our case would have to enclose the GPR antenna completely in order to be modeled, which may be many wavelengths in dimension. The second reason is that adding surface electric and magnetic currents as unknowns in standard MoM codes (such as the Numerical Electromagnetics Code (NEC) [12]) would require a significant amount of additional coding.

To circumvent these problems with direct techniques, we developed an iterative scheme that analyzes each subgeometry alternately, while sharing the surface current information between iterations. This scheme produces accurate results in surprisingly few iterations and demands only minimal changes in "standard" FDTD and MoM codes.

A flow chart of the iterative scheme is shown in Fig. 2. This scheme starts by using MoM to analyze the antenna geometry under the assumption that the initial electric and magnetic surface currents are zero. Evaluating the electric and magnetic fields just inside the Huygens' surface yields first-order estimates of the electric and magnetic surface currents $\boldsymbol{J}_{S 1}$ and $\boldsymbol{M}_{S 1}$, respectively. These currents are then used as source currents in an FDTD model of the ground-scatterer geometry. Second-order estimates $\boldsymbol{J}_{S 2}$ and $\boldsymbol{M}_{S 2}$ are then obtained by evaluating the magnetic and electric fields just outside the Huygens' surface. These second-order estimates can then be used as source currents (along with the voltage and/or current sources that drive the antenna directly) in a subsequent MoM analysis of the antenna geometry. Third-order estimates $\boldsymbol{J}_{S 3}$ and $\boldsymbol{M}_{S 3}$ can then be obtained by evaluating $\boldsymbol{E}$ and $\boldsymbol{H}$ just inside the Huygens' surface.

By continuing this procedure, the surface currents and fields in both subgeometries can be updated until they converge to their exact values. The iteration-to-iteration changes in the equivalent currents are due to the coupling between the antenna and the ground that is calculated with increasing accuracy as the iterative procedure progresses. Convergence can be checked either by comparing the fields or surface currents obtained after subsequent iterations or by testing to see that the null fields in either subgeometry are indeed negligible. Although we are not aware of a mathematical proof of convergence for this technique, our experience has shown it to be convergent for all cases we have encountered.

\section{IMPLEMENTING THE HYBRID TEChNique WITH STANDARD EM CODES}

A number of numerical techniques can be used to implement this hybrid technique as long as they are well suited to model the two subgeometries (the antenna and ground geometries). In this paper, MoM is chosen to model the antenna geometry because there is only a complex antenna in free space and no ground and no buried scatterers. FDTD is used to model 


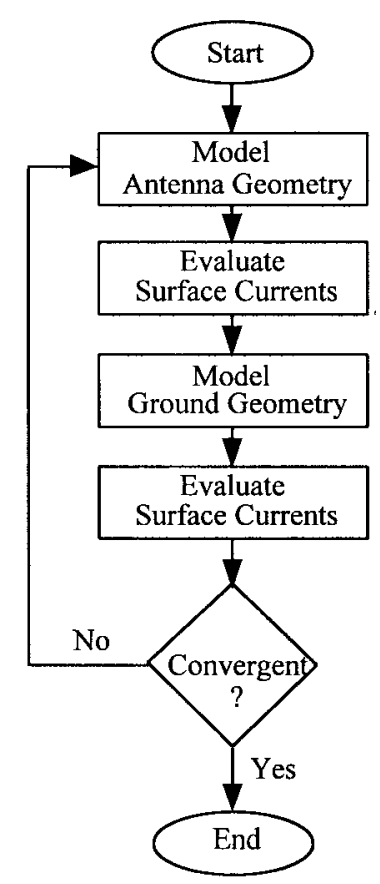

Fig. 2. Flow chart of the iterative procedure used in the hybrid technique.

the ground geometry, since no complex antenna is in this geometry.

Since FDTD is more restrictive spatially than MoM, the Huygens' surface is chosen to conform to the FDTD spatial lattice, with the sides lying along tangential $E$-field sample points. By doing this, the electric and magnetic surface currents also conform to the FDTD lattice.

In this section, the changes needed to use standard MoM and FDTD codes in the iterative scheme are outlined.

\section{A. Modifications to MoM}

Regardless of what integral equation is used and the kind of basis and testing functions chosen, an MoM formulation of an antenna or scattering geometry reduces to a system of equations of the form

$$
[Z][\boldsymbol{I}]=[V] .
$$

In this expression, the elements of impedance $[Z]$ are the reactions of the basis and testing functions, the unknown current vector $[I]$ contains the unknown basis-function coefficients, and the excitation vector $[V]$ contains the reactions between the testing functions on the scatterer and the source fields. These sources can either be lumped sources, such as voltage sources or incident fields from distant sources. Although a number of Green's functions can be used in conjunction with MoM, here we assume the use of the free-space Green's function, since it is assumed that the FDTD algorithm is responsible for accounting for the ground parameters.

When using an MoM code in conjunction with the iterative scheme, the equivalent electric and magnetic currents along the Huygens' surface are not treated as unknowns, since their values are determined via the previous iteration (i.e., from FDTD). Hence, these currents impact the solution via the excitation vector $[V]$. To accomplish this, all that is necessary is to calculate the reactions of the fields generated by the equivalent surface currents with the testing functions on the surface of the antenna and add these to contributions from the lumped sources on the antenna. This is a simple operation, since the surface currents can be approximated by a distribution of short filamentary currents on the rectangular grid, whose radiated fields are simple functions of position [12]. The fields generated by the surface currents can be obtained by superposition of the fields generated by all electric and magnetic filamentary-current moments on the Huygens' surface.

Once (3) has been solved for the current distribution on the antenna, updated electric and magnetic currents can be found simply by calculating the $E$ - and $H$-fields just inside the Huygens' surface and using (1) and (2), respectively.

\section{B. Modifications to FDTD}

The only change that must be made to a standard FDTD code in order to implement the hybrid scheme is the modifying of the field-advance equations to allow for the presence of the electric and magnetic surface currents along the Huygens' surface. The presence of the ground is modeled simply by specifying the ground-constitutive parameters in each cell. The bottom of the ground region can be closed numerically by using standard absorbing-boundary conditions, modified only by using the appropriate values of the ground permittivity [4].

In general, the equivalent currents cause all of the field components to exhibit discontinuities across the Huygens' surface. The way in which the field-advance equations need to be modified depends on whether the component in question is evaluated on or off the Huygens' surface.

Fig. 3(a) shows a portion of a Huygens' surface that lies in the $x z$ plane. For this case, the component $H_{y}$ is evaluated one half-cell to either side of the surface. Using Faraday's law to advance the component $H_{y}\left(i+\frac{1}{2}\right)$, we find

$$
-\oint_{C_{1}} \boldsymbol{E} \cdot d \boldsymbol{l}=\iint_{S_{1}} \boldsymbol{M}_{S} \cdot d \boldsymbol{s}+\mu \frac{\partial}{\partial t} \iint_{S_{1}} \boldsymbol{H} \cdot d \boldsymbol{s}
$$

where the contour $c_{1}$ extends up to but does not include the Huygens' surface [so there is no contribution to the magnetic surface-current integral on the right-hand side of (4)]. Even though $E_{z}$ is discontinuous at the Huygens' surface, the value $E_{z}^{+}(i)$ along the right-hand edge of the contour can be expressed in terms of the average value $E_{z}(i)$ at the surface using (2), which yields

$$
E_{z}^{n}\left(i, j, k+\frac{1}{2}\right)^{+}=E_{z}^{n}\left(i, j, k+\frac{1}{2}\right)+\frac{1}{2} M_{y}^{n}\left(i, j, k+\frac{1}{2}\right) .
$$

Similarly, the value $E_{z}^{-}(i)$ on the other side of the Huygens' surface is given by

$$
E_{z}^{n}\left(i, j, k+\frac{1}{2}\right)^{-}=E_{z}^{n}\left(i, j, k+\frac{1}{2}\right)-\frac{1}{2} M_{y}^{n}\left(i, j, k+\frac{1}{2}\right) .
$$

As a result, the only correction to the standard field-advance equation for $H_{y}$ on either side of the Huygens' surface is to replace $E_{z}$ with either (5) or (6), depending upon which side 


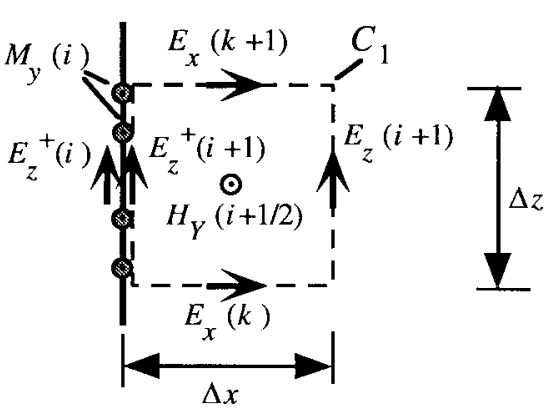

(a)

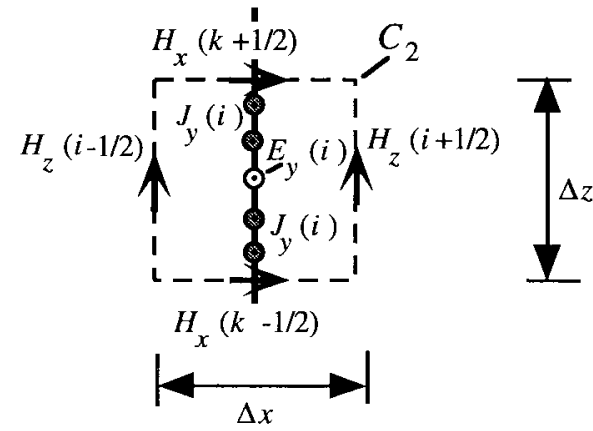

(b)

Fig. 3. (a) Integral contour for advancing the y component of the magnetic field at the right side of the $x z$ plane. (b) Integral contour for advancing the y component of the electric field on the $x z$ plane.

of the surface is being considered. The resulting field-advance equation for $H_{y}$ is

$$
\begin{aligned}
H_{y}^{n+(1 / 2)} & \left(i+\frac{1}{2}, j, k+\frac{1}{2}\right)-H_{y}^{n-(1 / 2)}\left(i+\frac{1}{2}, j, k+\frac{1}{2}\right) \\
= & \frac{\Delta t}{\mu}\left[\frac{E_{z}^{n}\left(i+1, j, k+\frac{1}{2}\right)-E_{z}^{n}\left(i, j, k+\frac{1}{2}\right)}{\Delta x}\right] \\
& -\frac{\Delta t}{\mu}\left[\frac{E_{x}^{n}\left(i+\frac{1}{2}, j, k+1\right)-E_{x}^{n}\left(i+\frac{1}{2}, j, k\right)}{\Delta z}\right] \\
& -\frac{\Delta t}{2 \mu \Delta x} M_{y}^{n}\left(i, j, k+\frac{1}{2}\right) .
\end{aligned}
$$

A similar correction can be derived for the component $H_{z}$. In (7), $E_{z}^{n}\left(i, j, k+\frac{1}{2}\right)$ is the average value of the electrictangential field on both sides of the magnetic-current sheet. When an equivalent electric-surface current $\boldsymbol{J}_{S}$ is present, the average electric-field value is calculated via the modified field-advance formula given in the next paragraph. A similar correction can be derived for the component $H_{z}$.

To update the average value of $E_{y}$ on the Huygens' surface, the integral form of Ampère's law is used

$$
\oint_{C_{2}} \boldsymbol{H} \cdot d \boldsymbol{l}=\iint_{S_{2}} \boldsymbol{J}_{S} \cdot d \boldsymbol{s}+\epsilon \frac{\partial}{\partial t} \iint_{S_{2}} \boldsymbol{E} \cdot d \boldsymbol{s}
$$

where the contour $c_{2}$ lies in the $x z$ plane and is shown in Fig. 3(b). Since this contour straddles the Huygens' surface, the electric-current integral will be nonzero whenever an equivalent current $J_{y}$ is present. Even though the component $H_{x}$ is discontinuous across the Huygens' surface, this discontinuity is automatically accounted for if the average value $H_{x}(i)$ is used in the expression. The same is true for $E_{y}$. Hence, the resulting field-advance equation for $E_{y}$ becomes (9), as shown at the bottom of the page. A similar correction can be derived for the component $\boldsymbol{E}_{z}$.

\section{Evaluating the Surface Currents}

Field information is exchanged between the antenna and the ground geometries via the electric and magnetic surface currents. At the end of each iteration, the tangential electric and magnetic fields are sampled at the Huygens' surface, and the resulting currents are then evaluated. In the case of the MoM iterations, the electric and magnetic fields are evaluated just inside the Huygens' surface at points corresponding to FDTD tangential $E$-field points. From these, $\boldsymbol{J}_{S}$ and $\boldsymbol{M}_{S}$ are found using (1) and (2), respectively.

In order to obtain the correct electric and magnetic currents at the end of each FDTD iteration, the discontinuities of these fields across the Huygens' surface must be accounted for, remembering that the $E$-field and $H$-field values calculated by FDTD at the boundaries are average values. The tangential $E$ fields just outside the Huygens' surface can be obtained from the average values using either (5) or (6), depending on whether the evaluation point is to the right or left of the surface. The tangential magnetic fields must be determined at the same points. However, since the magnetic and electricfield points are interlaced, $H$-field values on both sides of the Huygens' surface must first be averaged. The discontinuity due to the currents can then be accounted for using equations similar to (5) and (6).

Since FDTD and MoM work in the time and frequency domains, respectively, it is necessary to transform the electric and magnetic currents from one domain to the other at the end

$$
\begin{aligned}
E_{y}^{n}(i, j & \left.+\frac{1}{2}, k\right)-E_{y}^{n-1}\left(i, j+\frac{1}{2}, k\right) \\
= & \frac{\Delta t}{\epsilon}\left[\frac{H_{x}^{n-(1 / 2)}\left(i, j+\frac{1}{2}, k+\frac{1}{2}\right)-H_{x}^{n-(1 / 2)}\left(i, j+\frac{1}{2}, k-\frac{1}{2}\right)}{\Delta z}\right] \\
& -\frac{\Delta t}{\epsilon}\left[\frac{H_{z}^{n-(1 / 2)}\left(i+\frac{1}{2}, j+\frac{1}{2}, k\right)-H_{z}^{n-(1 / 2)}\left(i-\frac{1}{2}, j+\frac{1}{2}, k\right)}{\Delta x}\right]-\frac{\Delta t}{\epsilon \Delta x} J_{y}^{n-(1 / 2)}\left(i, j+\frac{1}{2}, k\right) .
\end{aligned}
$$




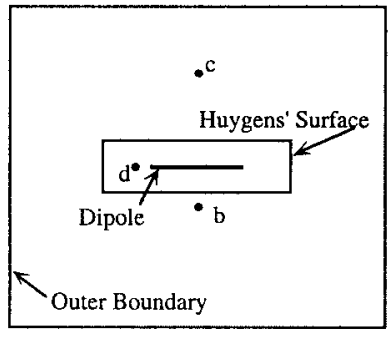

(a)

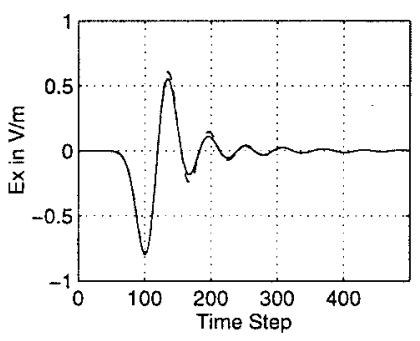

(c)

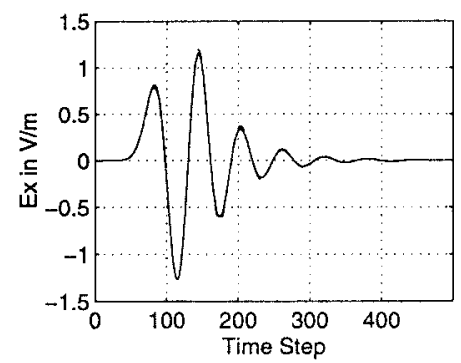

(b)

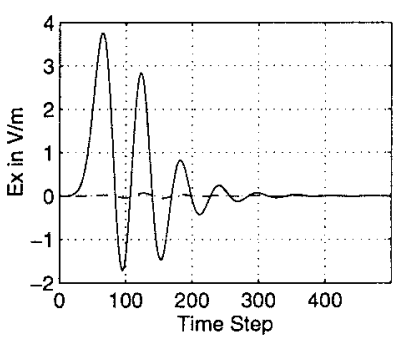

(d)
Fig. 4. Thin wire dipole in free space. (a) Geometry, (b) and (c) Electric field $E_{x}$. (b) is $0.4 \mathrm{~m}$ and (c) is $0.8 \mathrm{~m}$ from the dipole center with solid curves calculated by FDTD and dotted curves calculated by the FDTD/MoM hybrid technique. (d) Electric field $E_{x}$ from dipole end, inside Huygens' surface, with solid curve representing actual field calculated by FDTD and the dotted curve representing the field radiated by Huygens' currents.

of each iteration. This is accomplished by using a Fast Fourier Transform (FFT) routine.

\section{NUMERICAL ResUlts}

\section{A. Dipole in Free Space}

The modifications to the FDTD equations needed for modeling the surface currents are an important part of the hybrid technique. To check the accuracy of these modifications, we first chose to model a dipole in free space aligned along the FDTD lattice. Such simple geometry can be modeled either by FDTD alone or with the iterative procedure, using FDTD for both the interior and exterior geometries. The iterative procedure for this case should produce an exact result after just one cycle, since the exterior geometry contains only free space.

The dipole is a thin, 0.6-m-long wire along the $\mathrm{x}$ axis, driven by a 6-ns, double-peak Gaussian-pulse voltage source. The solution space is a $70 \times 50 \times 70$-cell FDTD space, with a cell size of $0.04 \mathrm{~m}$. The Huygens' surface is $30 \times 10 \times 10$ cells, symmetrically surrounding the dipole.

Fig. 4(a) shows the geometry for this case. Fig. 4(b) and (c) each show the field $E_{x}$ as calculated by FDTD alone (solid curves) and by the FDTD/MoM hybrid technique (dotted curves) at distances 0.4 and $0.8 \mathrm{~m}$ from the dipole center, respectively. These curves show excellent agreement between the two techniques. The difference between them is less than $-30 \mathrm{~dB}$. Fig. 4(d) shows the field $E_{x}$ a distance $0.2 \mathrm{~m}$ from the dipole tip. The solid curve shows the actual field, calculated by FDTD alone. The dotted curve shows the field radiated

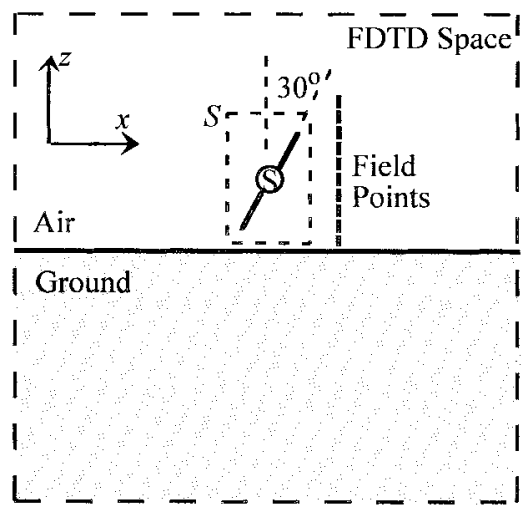

Fig. 5. Skewed dipole above a homogeneous ground with $\epsilon_{r}=4$ and $\sigma=0$.

by the Huygens' currents alone. Since this point is inside the Huygens' surface, this curve is nearly zero, as it should be.

\section{B. Skewed Dipole Above a Homogeneous Ground}

For a simple verification of the full-hybrid technique, consider the geometry shown in Fig. 5, which shows a skewed dipole consisting of a thin wire $(0.6 \mathrm{~m}$ long) offset from the $z$ direction by $30^{\circ}$. The center of the dipole is $0.56 \mathrm{~m}$ above a ground that has relative permittivity of 4.0 and is lossless. The dipole is driven at its center by a continuous wave-voltage source at a frequency of $150 \mathrm{MHz}$.

To model this geometry using the hybrid technique, we chose the Huygens' surface to be a $0.4 \times 0.4 \times 1.2-\mathrm{m}$ volume, symmetrically surrounding the dipole. For the ground geometry, we used a $70 \times 50 \times 70$-cell numerical lattice for FDTD. We also used the Numerical Electromagnetics Code (NEC) [12], a widely used MoM code, to model the antenna geometry. The antenna-voltage feed was a 1.5-ns Gaussian pulse. The FDTD calculations were computed out to $40 \mathrm{~ns}$ so as to adequately take into account multiple interactions between the ground and the antenna.

The dotted curves in Fig. 6 are the $z$ component of the electric field on a $z$-directed line, $0.32 \mathrm{~m}$ away from the antenna (calculated using the hybrid technique at three different iterations). The solid curve is the result calculated using NEC alone, which is capable of singlehandedly modeling the fields above (but not beneath) the ground for this geometry. These curves show that the fields calculated by the hybrid technique do indeed converge to the correct result.

Fig. 7 shows the calculated antenna-current distribution as a function of the iteration number. Also shown is the result calculated by the NEC code. As can be seen, the iterativetechnique results converge quickly. Since the ground in this case was lossless, the effect on the antenna-current distribution was not large. Even so, it is evident from Fig. 7 that the ground has forced a slight asymmetry of the current, as would be expected.

\section{Bow-Tie Antenna Above a Stratified Ground}

A final experiment that shows the type of complex geometries that can be modeled by this iterative technique 


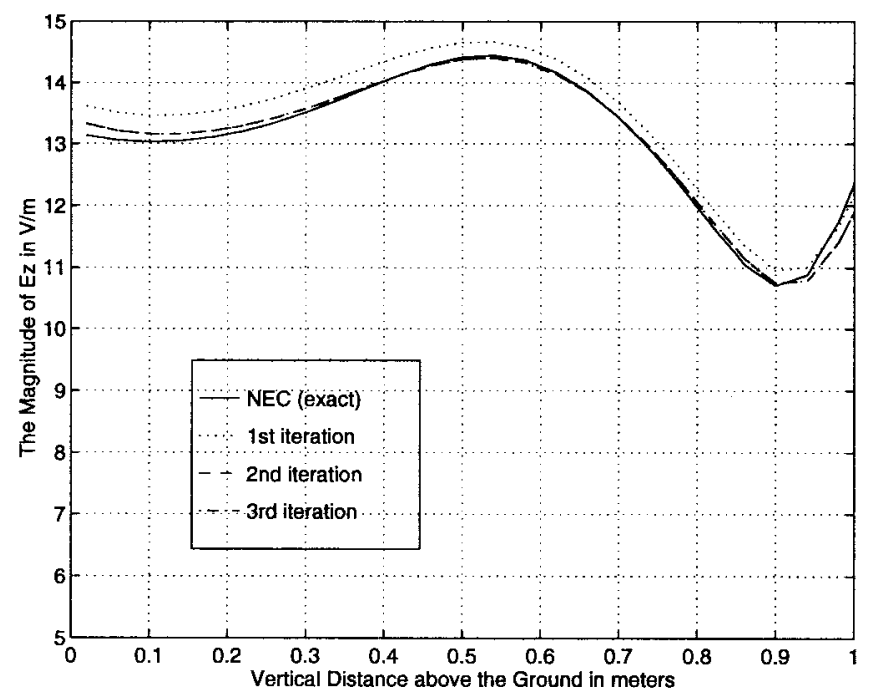

Fig. 6. Electric fields radiated by the skewed dipole, at $0.32 \mathrm{~m}$ away from the dipole along the $z$ direction. The MoM result is evaluated by using NEC-2, and the hybrid results are evaluated using an FDTD/MoM combination.

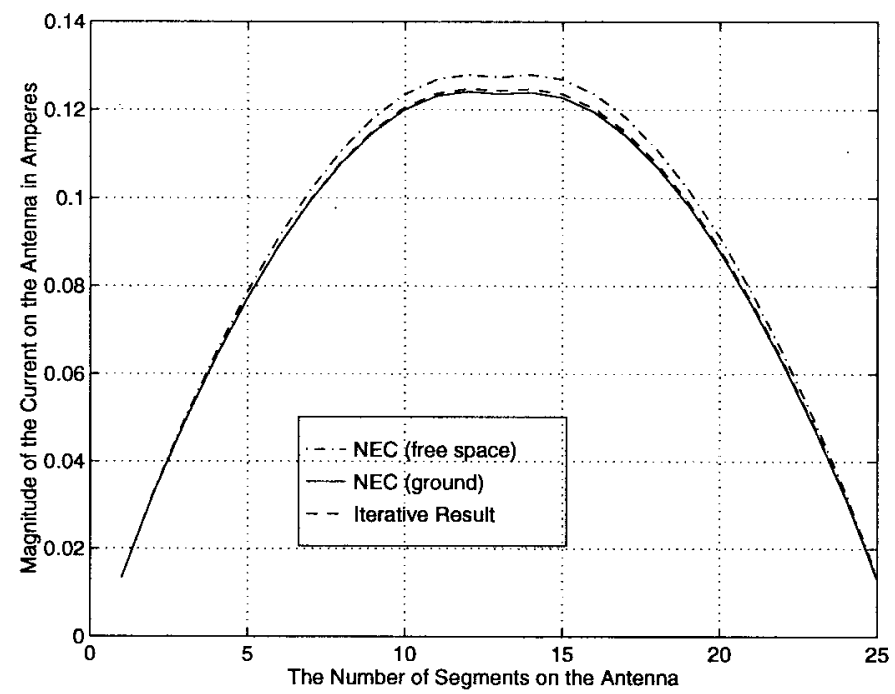

Fig. 7. Current distribution on the skewed dipole, as calculated by the NEC code in free space, the NEC code with the ground present, and the FDTD/MoM hybrid technique.

is shown in Fig. 8. Here, a bow-tie antenna is positioned above a stratified ground. The bow-tie antenna consists of two equilateral triangles with $0.22-\mathrm{m}$ sides made of thin metal wires. The antenna is driven by a $150-\mathrm{MHz}$ voltage source at its center and is located $0.4 \mathrm{~m}$ above the ground. The ground is complex, consisting of 30 layers, each with a thickness of $0.04 \mathrm{~m}$ (the deepest layer extends to infinity). The relative permittivity of each layer varies linearly with depth from 3.0 to 6.0 , and the conductivity of each layer is $0.001 \mathrm{~S} / \mathrm{m}$. The stratified ground is modeled using the formalism found in [6]. The FDTD space is $70 \times 50 \times 70$ cells, and the Huygens' surface is a $30 \times 10 \times 10$-cell rectangle $(1.2 \times 0.4 \times 0.4 \mathrm{~m})$ that surrounds the bow-tie antenna symmetrically. Since the round-trip time from the antenna to the bottom-ground strata was roughly $20 \mathrm{~ns}$, performing the FDTD calculations out to

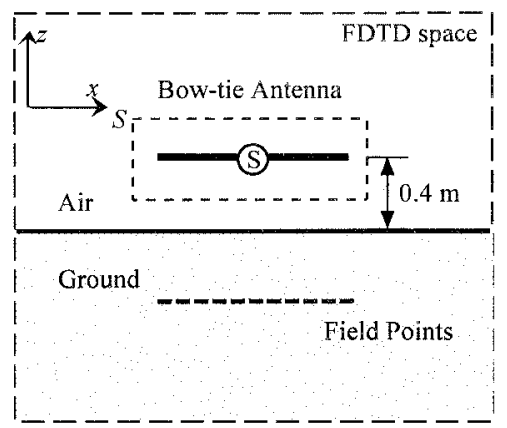

Top view of Bow-tie Antenna

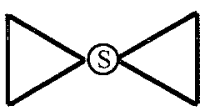

Fig. 8. Bow-tie antenna above a stratified ground. Each strata is 0.04 thick, and $\epsilon_{r}$ varies linearly from 3.0 at the top to 6.0 at depths of $12 \mathrm{~m}$ (and below). $\sigma=0.001 \mathrm{~S} / \mathrm{m}$ for all strata.

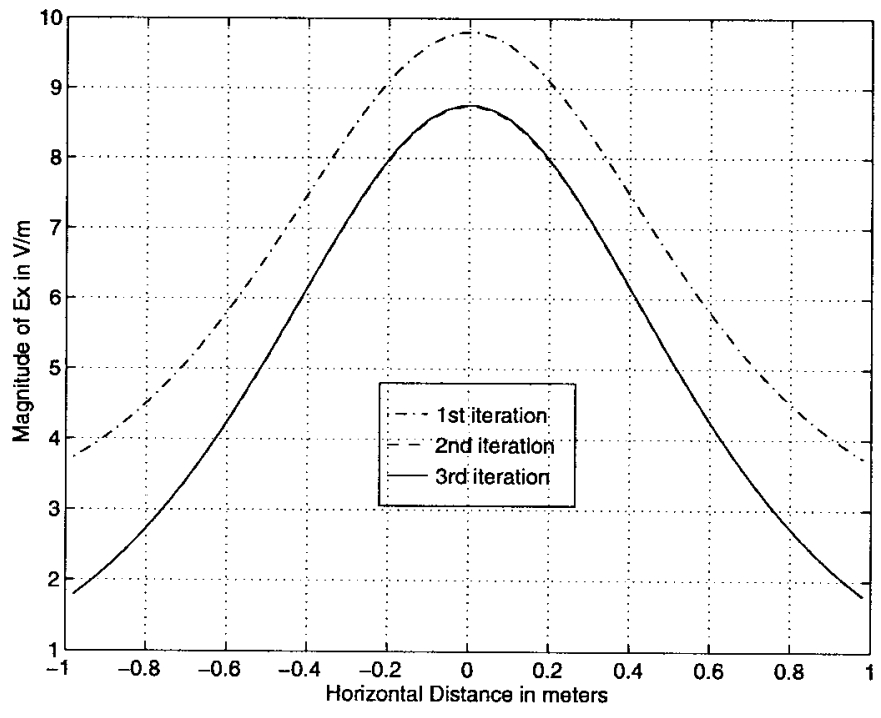

Fig. 9. Magnitude of the $x$ component of the electric field, radiated by the bow-tie antenna, sampled at $0.4 \mathrm{~m}$ under the ground, along the $x$ direction. Results are evaluated by the FDTD/MoM hybrid method.

$40 \mathrm{~ns}$ was adequate to model the multiple reflections between the antenna and the ground.

The iterative procedure was run for three full iterations. A convergent result was obtained at the end of the second iteration. Fig. 9 shows the magnitude of $E_{x}$, sampled 0.4 $\mathrm{m}$ under the ground (directly under the antenna) after two iterations. Here, the field level changed markedly between the first and second iterations, since the ground in this case was lossy.

\section{Computational Requirements}

Our experience has shown that this iterative scheme typically produces useful results after one complete iteration (i.e., one interior geometry and one exterior geometry calculation) and essentially exact results after two complete iterations. When using MoM for the antenna geometry and FDTD for the ground geometry, the computational requirements for each MoM calculation were usually negligible compared to those required for each FDTD calculation.

Given this, it generally can be said that the computational cost of the iterative technique is roughly twice the cost of a comparable FDTD simulation that uses the same cell size. 


\section{CONCLUSIONS}

We have presented a hybrid technique for modeling complex antennas in the presence of heterogeneous grounds. This technique allows two dissimilar electromagnetic-numerical methods to be used to model different aspects of a complex geometry. It is easy to implement with MoM and FDTD but also possible with other suitable numerical methods. This technique also provides a less-expensive way of characterizing an antenna above an inhomogeneous ground, because it avoids the expensive evaluation of the Green's function. Numerical simulations of modeling complex GPR antennas have been performed on common workstations or desktop PC's.

This hybrid technique greatly increases the range of antennas that can be modeled in the presence of a ground by using ordinary electromagnetic codes that are well suited to the characteristics of the antenna in free space instead of heterogeneous ground. Furthermore, the hybrid technique can easily be extended to model multiple-antenna GPR's by setting up multiple-antenna subgeometries. Antenna modeling is usually the weak link of most numerical GPR modelers, so this hybrid technique should greatly enhance the modeling of real GPR's.

\section{REFERENCES}

[1] K. S. Yee, "Numerical solution of initial boundary value problems involving Maxwell's equations in isotropic media," IEEE Trans. Antennas Propagat., vol. AP-14, pp. 302-307, Apr. 1966.

[2] J. M. Bourgeois and G. S. Smith, "Full electromagnetic simulation of a ground penetrating radar: Theory and experiment," Proc. IEEE Antennas and Propagation Int. Symp., Seattle, WA, vol. 3, pp. 1442-1443, June $19-24,1994$.

[3] R. L. Roberts and J. J. Daniels, "Finite difference time domain forward modeling of GPR data," Proc. 5th Int. Conf. Ground Penetrating Radar, Kitchener, Ont., Canada, 1994, pp. 185-204.

[4] K. R. Demarest, Z. Huang, and R. G. Plumb, "An FDTD near-to far-zone transformation for buried scatterers," IEEE Trans. Antennas Propagat., vol. AP-44, pp. 1150-1157, Aug. 1996.

[5] Z. Huang, "A numerical ground-penetrating radar simulator," $\mathrm{Ph} . \mathrm{D}$ dissertation, Univ. Kansas, Lawrence, 1996.

[6] K. R. Demarest, R. G. Plumb, and Z. Huang, "FDTD modeling of scatterers in stratified media," IEEE Trans. Antennas Propagat., vol. AP-43, pp. 1164-1168, Oct. 1995.

[7] K. L. Shlager, G. S. Smith, and J. G. Maloney, "Optimization of bowtie antennas for pulse radiation," IEEE Trans. Antennas Propagat., vol. AP-42, pp. 975-982, July 1994

[8] J. M. Bourgeois and G. S. Smith, "A fully three-dimensional simulation of a ground-penetrating radar: FDTD theory compared with experiment," IEEE Trans. Geosci. Remote Sensing, vol. 34, pp. 36-44, Jan. 1996.

[9] R. F. Harrington, Field Computation by Moment Methods. New York: Macmillan, 1968.

[10] , Time-Harmonic Electromagnetic Fields. New York: McGrawHill, 1961.

[11] R. F. Harrington and J. R. Mautz, "A generalized network formulation for aperture problem," IEEE Trans. Antennas Propagat., vol. AP-24, pp. 870-873, Nov. 1976.

[12] G. J. Burke and A. J. Pogio, Numerical Electromagnetics Code (NEC)Method of Moments, Tech. Doc. 116, Naval Ocean Syst. Center, San Diego, CA, Jan. 1981.

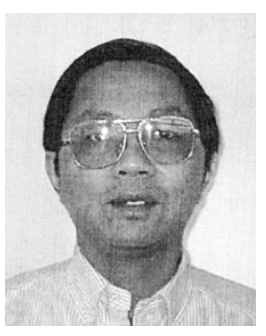

Zhubo Huang (S'93-M'96) was born in Shanghai, China, in 1962. He received the B.S. degree in physics from East China Normal University, Shanghai, in 1982, the M.S. degree in electrical engineering from Beijing University of Aeronautics and Astronautics through a cooperative program with Beijing Institute of Remote Sensing Equipment, and the Ph.D. degree in electrical engineering from the University of Kansas, Lawrence, in 1996.

From 1984 to 1992, he was an Engineer with the Beijing Institute of Remote Sensing Equipment. His main research areas were radomes, antennas, design of radome/antenna automatic measurement systems and engineering applications of microwave technology. From 1992 to 1996, he was a Graduate Research Assistant in the Radar Systems and Remote Sensing Laboratory, University of Kansas. Since 1996, he has been Senior Planner with Sprint PCS, Kansas City, MO. His current research interests are computational electromagnetics, groundpenetrating radar simulation, antennas, RF technology, CDMA customer premises equipment, system and technology integration, and wireless data services

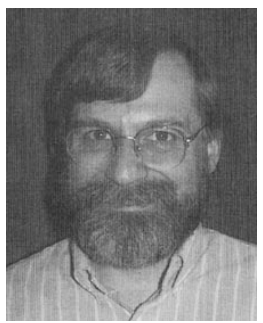

Kenneth R. Demarest (S'78-M'79-SM'98) was born in Hackensack, NJ, on December 16, 1952. $\mathrm{He}$ received the B.S. degree in electrical engineering from John Brown University, Siloam Springs, AK, on 1974, and the M.S. and Ph.D. degrees in electrical engineering from The Ohio State University (OSU), Columbus, in 1976 and 1980, respectively.

From 1974 to 1979 , he was associated with the ElectroScience Laboratory, OSU, as a Graduate Research Associate. From 1979 to 1984, he was an Assistant Professor in the Electrical Engineering Department, Lafayette College, Easton, PA. Since 1984, he has been with the Electrical Engineering and Computer Science Department, University of Kansas, Lawrence, most recently as a Professor. His research interests are in the areas of fiber optic comuunications and electromagnetics. He is the author of a number of papers, book chapters, and the book Engineering Electromagnetics.

Dr. Demarest is a member Eta Kappa $\mathrm{Nu}$ and the International Union of Radio Science Commission B.

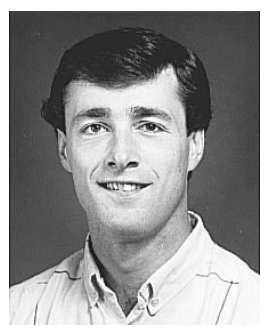

Richard G. Plumb (S'82-M'88-SM'94) was born in Syracuse, NY in 1959. He received the B.S., M.S., and Ph.D. degrees in electrical engineering from Syracuse University in 1982, 1985, and 1988 , respectively.

From 1982 to 1986, he worked at General Electric, Syracuse. In 1985, he graduated from General Electric's Advanced Course in Engineering. Between 1986 and 1988, he was a Graduate Research Assistant with Syracuse University, and for the 1988 academic year, he was a Visiting Assistant Professor. In 1989, he joined the faculty in the Department of Electrical Engineering and Computer Science, University of Kansas, Lawrence. In 1996, he was a Visiting Scholar at the University of Queensland, Brisbane, Australia. In 1998, he joined the Electrical Engineering Department, State University of New York, Binghamton, where he is currently Professor and Chair. His primary research interests are in electromagnetics, ground-penetrating radar, and inverse scattering. 